\title{
APPLICATION OF A MODEL-DRIVEN APPROACH TO THE DEVELOPMENT OF DISTRIBUTED SIMULATIONS: THE ESA HRAF CASE
}

\author{
Andrea D'Ambrogio \\ Paolo Bocciarelli \\ Department of Enterprise Engineering \\ University of Rome Tor Vergata \\ Via del Politecnico, 1 \\ 00133 Rome, Italy \\ \{bocciarelli,dambro\}@uniroma2.it
}

\author{
Juan Delfa \\ ESA-ECSAT \\ Harwell Campus \\ Didcot, Oxfordshire \\ OX11 0FD, UK \\ Juan.Delfa@esa.int \\ AT
}

\author{
Aron Kisdi \\ GMV \\ Harwell Campus \\ Didcot, Oxfordshire \\ OX11 0QG, UK \\ akisdi@gmv.com
}


Due to the a lack of critical infrastructures that would allow the $\mathrm{V} \& \mathrm{~V}$ of autonomy components at mission level, the European Space Agency (ESA) started the MREP-2 (Mars Robotic Exploration Preparation-2) programme, whose main objective is the specification and implementation of the HRAF (Harwell Robotics and Autonomy Facility) HLA DS (Distributed Simulation) EF (Engineering Framework), an architectural framework which aims at supporting integration and $V \& V$ of autonomy systems and associated technologies from unit up to mission level, based on the High Level Architecture (HLA) standard (IEEE 2010b).

In this context, this paper presents the result of an ESA founded research and development activity that aims at investigating how a model-driven approach can be effectively exploited to support the objective of the HRAF project.

The contribution proposed in this paper achieves two main objectives:

- according to a methodological perspective, this work introduces a development process consisting of a DSEEP (Distributed Simulation Engineering and Execution Process) standard (IEEE 2010a) extension, which exploits standards and tools introduced by the Model Driven Architecture (MDA) (OMG 2014). The specification of the development process also addresses the identification of the relevant tool chain.

- according to an implementation perspective, this work presents the software artifacts developed for supporting the proposed model-driven HLA-DS Engineering Framework, namely:

(i) the Model-To-Text Trasformation Tool (M4T), which implements a model transformation for generating the Federation Object Model (FOM) used to describe the information shared by distributed components in a HLA-based simulation (IEEE 2010c);

(ii) the FOM-HLA Profile, for annotating the mission-level abstract specification of the federation under study, i.e, a SysML model constituting the main input to M4T;

(iii) the SpaceFOM Data Types Library, an external library referenced by the SysML federation model, to ensure compliance with the SISO Space Reference FOM (Möller et al. 2016), according to the HRAF requirements.

The reminder of this paper is structured as follows. Section 2 summarizes related work. Section 3 recalls the abstract DSEEP tailoring, as proposed in (Bocciarelli et al. 2019). Section 4 illustrates an overview on the ESA HRAF project and outlines the addressed operational scenarios. Section 5 describes how the abstract DSEEP tailoring has been adapted to fit the specific HRAF HLA-DS EF needs. This section also describes the software components that are part of the proposed framework implementation. Section 6 discusses the actual application of the HRAF framework to the scenarios addressed by the HRAF project, and Section 7 gives concluding remarks.

\section{RELATED WORK}

A large effort has been spent over the past years to address the development of distributed simulations. As such topic has been faced from various perspectives, the literature review hereby proposed has focused on those contributions dealing with the introduction of model-driven methodologies, tools and frameworks for easing the distributed simulation design and implementation activities.

The adoption of a model-driven paradigm to mitigate the several difficulties that typically affect the effective adoption of distributed simulation in real systems engineering projects has been investigated in (Bocciarelli et al. 2012, Bocciarelli et al. 2015, Kapos et al. 2014, Jain et al. 2015, Topçu et al. 2016). This paper is founded on such contributions and proposes an approach that introduces model-driven standard and technologies to support ESA in developing distributed simulations, by automating the generation of the 
distributed simulation object model, according to the requirements of the Harwell Robotics and Autonomy Facility space exploration programme.

In (Cetinkaya et al. 2011), a model driven simulation model development life-cycle which exploits author's past contributions is presented, along with a supporting framework based on a chain of model transformations. In (Özhan and Oguztüzün 2015), a MDA-based method to map a field artillery conceptual model to an HLA federation architecture model is proposed. The method also includes a code generator that takes as input the federation model, and produces as output the source code that can be executed on a runtime infrastructure.

Similarly to the aforementioned contributions, the first issue this work deals with is the specification of a development process, which might effectively help to identify and carry-out the several activities required for building a distributed simulation. In this respect, the methodology proposed in this work is based on the DSEEP, which has been extended so as to benefit from the adoption of standards and tools introduced by the MDA. The proposed tailoring is a refinement of previous work (Bocciarelli et al. 2019). As regards the implementation view point, it should be noted that the objective of this work doesn't consist in the development of a full-fledged supporting tool, as ESA is already adopting a set of tools which conform the corporate best practice and guidelines (i.e., Magic Draw for model development, Pitch Developer Studio as the HLA-based distributed environment development, etc.). The main objective of this paper is instead the development of a set of software artifacts (i.e, UML Profiles, SysML models and model transformations) so as to effectively enable the use of the proposed DSEEP-based methodology, using the toolchain currently adopted by ESA.

\section{BACKGROUND}

The proposed Framework is founded on a model-driven development process, which in turn has been defined as an extension of DSEEP, i.e., the standard defining a process model for DS development and execution.

In (Bocciarelli et al. 2019) we proposed a general model-driven method that aims at automatically generating a FOM from an abstract SysML model representing a federation. Such a method has been obtained by tailoring the DSEEP according to the following requirements:

- the method shall support the adoption of model-driven standards so as to support the automated generation of FOM modules;

- the tailoring shall be based on a clear separation of the domain model representation from the concepts and data types specified by a reference FOM;

- the method shall be flexible enough not to be tied to the reference FOM adopted in the HRAF project;

In order to meet the above-mentioned design guidelines, the proposed method exploits two different software artifacts: a Data Types Library, which provides a SysML representation of data types and objects specified in a given reference FOM, and a Profile, which allows one to annotate the input SysML model so as to include HLA-related marks.

The so obtained method constitutes one of the starting points for the specification of the model-driven process proposed in this paper. For a detailed description of the first abstract tailoring of the DSEEP, interested readers are referred to (Bocciarelli et al. 2019).

Such a tailored DSEEP process is hereby adapted to meet the operational requirements of the HRAF project, as discussed in 5.1. 


\section{ESA HRAF PROJECT OVERVIEW AND SCENARIOS}

The HRAF programme has followed an incremental development strategy. While the former HRAF Pilot 1 and Pilot 2 phases addressed the definition of the Core Architecture and discussed the related validation and verification through specific demonstration cases, the current Pilot 3 phase (HRAF Exploration) deals with the development and validation of a model-driven HLA Distributed Simulation (HLA-DS) Engineering Framework for supporting the semi-automated generation of the Federation Object Model (FOM) in the space domain.

Specifically, the activity discussed in this paper is part of the HRAF Pilot 3, whose main objective is twofold. The first group of tasks aims to extend the space domain application of the HRAF HLA-DS with a planetary entry, descent and landing system. The second group of tasks focuses on the development of a Modeldriven HLA-DS Engineering Framework for supporting the (semi-)automated generation of the FOM for each space domain federate in HRAF.

As outlined in Section 1, and better discussed in the next section, this work addresses the second group of activities. In this respect, as a part of the HRAF exploration project objective, the Model-driven HLA-DS Engineering Framework has been applied to two different scenarios.

The first one, named Autonomous Rendezvous and Capture in Mars Orbit, is structured in the missions Mars Sample Return (MSR) and Earth Return Orbiter (ERO), which share the common objective of returning to Earth a set of rigorously documented Mars atmospheric, soil, and rock core samples before the end of 2029.

The second scenario, named Low-Gravity Body Descent and Precision Landing, focuses on an asteroid targeting, descent, landing and sampling operation. This scenario includes the investigation of a NEO (Near Earth Object) Craft and the related GNC (Guidance, Navigation and Control) system, as well.

\section{MODEL-DRIVEN HLA-DS ENGINEERING FRAMEWORK}

The HRAF HLA-DS EF design is based on the abstract DSEEP extension discussed in Section 3 and adapted in this work so to make it compliant with the specific HRAF operational requirements. The main objective of the development process is to support the transition from a functional architecture to the software implementation and the related system integration, by introducing simulation techniques.

More specifically, the model-driven framework allows one to obtain a DS of the space system under study, starting from an abstract functional architecture specified in SysML, the reference modeling language in the systems engineering context (OMG 2017).

The model-driven framework makes use of different tools, ranging from commercial products to open-source tools and ad-hoc systems specifically designed and implemented to meet the HRAF Pilot 3 requirements. Subsection 5.1 provides a detailed understanding of both the activities composing the development process and the related tool chain, while Subsection 5.2 and Subsection 5.3 describe the UML Profile and the Datatype Library at the basis of the proposed framework implementation, respectively.

\subsection{Model-driven Method and Tool Chain}

The Engineering Framework enables a designer to first create a SysML model representing the federation under study and then use the SysML model for generating the set of HLA FOM modules which describe the data exchanged during the simulation. The FOM modules are in turn used for generating the skeleton of the several simulation components (or federates) implementing the HLA simulation (or federation).

Figure 1 illustrates the process that extends the abstract DSEEP tailoring discussed in Section 3. 


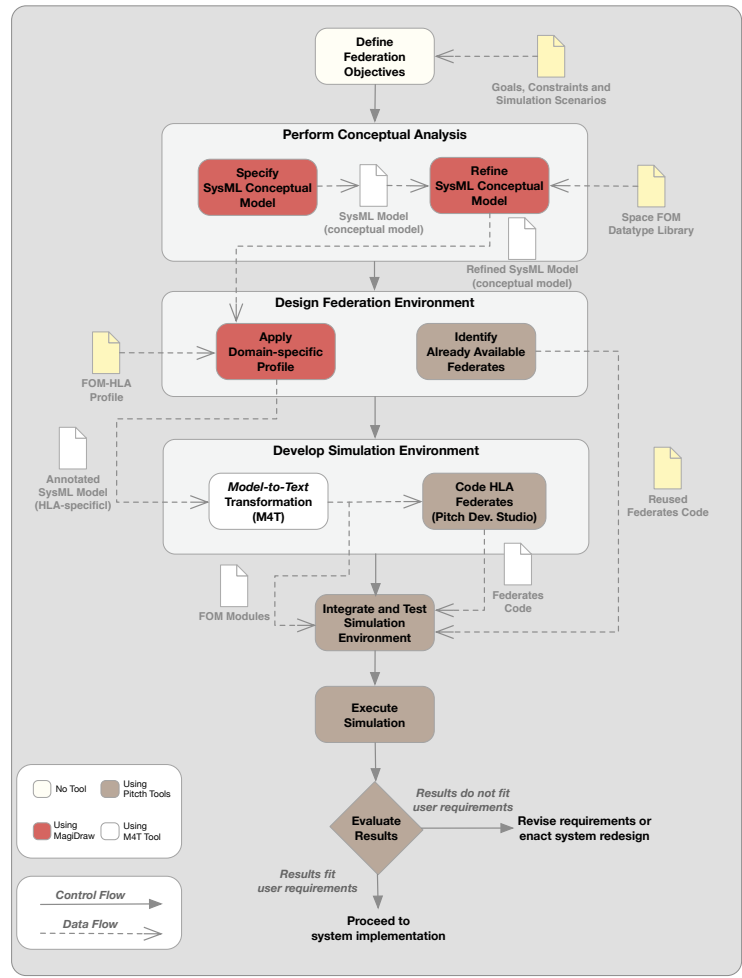

Figure 1: Tailored DSEEP.

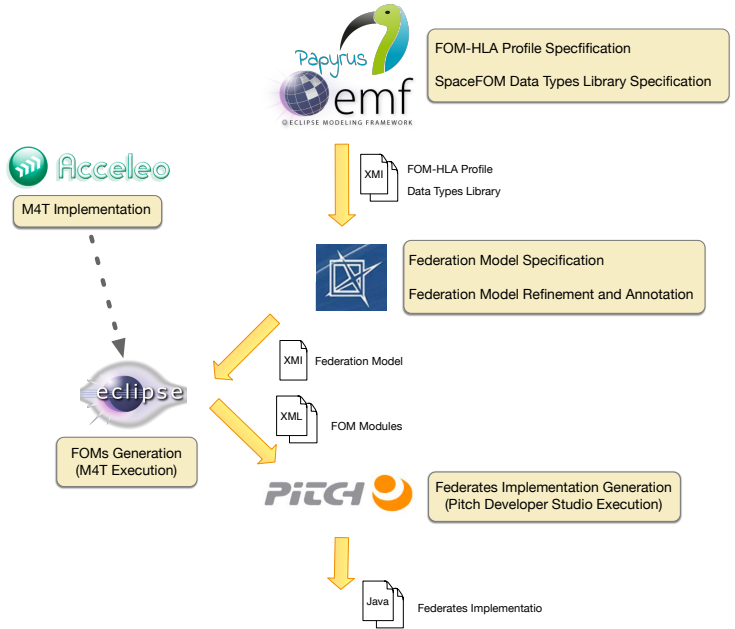

Figure 2: HRAF HLA-DS Toolchain.

One of the pillars at the basis of the method specification is the compliance to any given FOM reference model. In this respect, in order to keep the method general enough to be used in different domains and to ensure a clear separation of the domain model representation from the concepts and data types specified by a reference FOM two different software artifacts are introduced:

- A FOM-HLA profile, which allows one to annotate the SysML model with FOM and HLA concepts;

- A Data Types Library, which provides a SysML representation of data types and the objects hierarchy specified in the reference FOM.

According to Figure 1, the proposed development process is structured as follows:

1. Define Federation Objectives: this step deals with the identification of the simulation objectives and constraints, together with the specification of the addressed scenario

2. Perform Conceptual Analysis: once the federation objectives have been clearly identified, a SysML conceptual model of the Federation is initially specified. Such a model contains elements representing the several entities that participate to the federation, which, at this level of abstraction, does not include any concepts related to the used middleware (e.g., HLA) or any references to concepts and data types related to a given Reference Domain. Then, a refinement step is also enacted in order to make the federation model compliant to the Space Reference FOM by using the Space FOM Data Types Library (introduced in Section 5.3).

3. Design Federation Environment: this step consists of two different activities. The first one further refines the federation model by applying the FOM-HLA Profile, so as to annotate those model ele- 
ments playing a role in terms of of HLA concepts (e.g., a federate, an object attribute, etc.). Then, the second activity verifies, for each identified federate, the availability of existing implementations.

4. Develop Simulation Environment: similarly to the federation design, the implementation of the federation environment consists of two sub steps. At the first one, $M 4 T$ is used to generate a set of FOM modules from the annotated SysML representing the Federation. At the second step, the Pitch Developer Studio commercial tool is used to generate the skeleton of the required federates, starting from the related FOM modules.

5. Integrate and Test Simulation Environment: during the integration step, the actual implementation of the federation is finalized. The implementation of the ad-hoc federates generated at the previous step is completed, and the set of existing federates are fine-tuned so as to be compliant with the rest of the federation implementation.

6. Execute Simulation: in this step the DS is deployed and executed.

7. Evaluate Results: in the last step, the obtained output is finally analysed in order to evaluate the potential need of a redesign activity or a requirements revision.

As the method includes several steps accomplished by different partners, each step requiring the use of a specific tool for being executed, a relevant issue faced in this project is the identification of both the most effective tool chain and the most suitable interchange format, so to ensure a seamless artifacts exchange among different partners. The proposed method requires the availability of a concrete implementation of the MDA standard for specifying metamodels, models, profiles and model-to-text transformations. In this respect, this project makes use of the Eclipse framework and its relevant plugins. Some commercial tools, which are part of the currently adopted ESA tool chain, are also used.

The complete tool chain is depicted in Figure 2, and includes the following tools:

- Eclipse Modeling Framework (EMF), which provides an implementation of the MDA standards used in this project;

- Acceleo (Eclipse Foundation 2015), as an implementation of the MOFM2T (OMG 2008), for implementing the model-to-text transformation engine of M4T;

- Papyrus (Eclipse Foundation 2019), the Eclipse Plugin dealing with UML and SysML modeling, which in this project has been used for specifying the FOM-HLA Profile and the Data Types Library;

- Magic Draw (NoMagic 2019), the commercial tool used in ESA for the specification and the refinement of the SysML federation model;

- M4T, which implements the model-to-text transformation engine for generating the set of FOM modules from the SysML model;

- Pitch Developer Studio (Pitch 2019) which is the commercial tool used in ESA for handling FOMs and supporting federates implementation.

Finally, two standards are used to enable tools interoperability: i) Ecore, the Eclipse EMF language for specifying metamodels, and ii) XML Metadata Intercharge (XMI) (OMG 2015), the MDA standard for the serialization and the interchange of models.

\subsection{FOM-HLA Profile}

The FOM-HLA profile has been introduced in (Bocciarelli et al. 2019) and, as stated in Section 1, its main objective is to extend the expressiveness of the Federation model in order to associate SysML elements to HLA-related concepts and, ultimately, to drive the subsequent model-to-text transformation in charge of generating (part of) the actual federation implementation. In this work, such a preliminary version of the 
FOM-HLA profile has been revised and extended in order to better comply with the HLA Object Model Template specification. The proposed extension mainly addresses the Identification table and the Datatypes table. Specifically, the FOM-HLA Profile includes a finer representation of complex datatypes (e.g., enumerations and arrays), as depicted in Figure 3 and Figure 4.

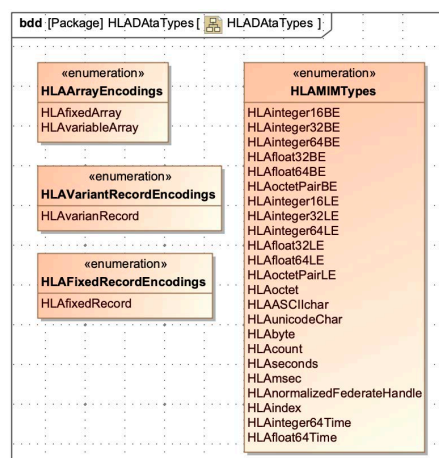

Figure 3: FOM-HLA Profile extension: complex datatypes.

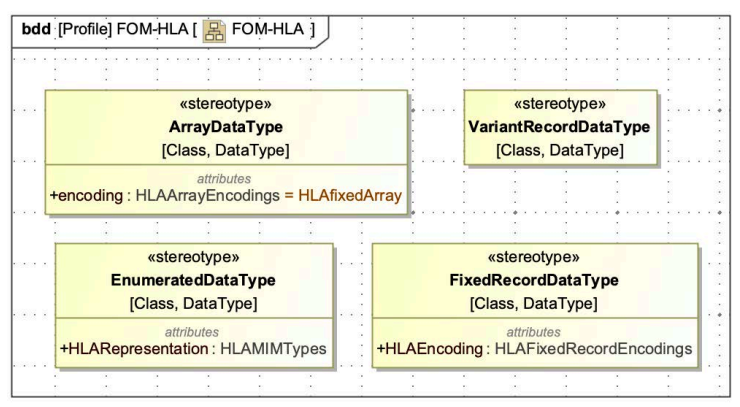

Figure 4: FOM-HLA Profile extension: stereotypes.

\subsection{Space FOM Datatypes Library}

Among the requirements at the basis of the HRAF DS Enginering Framework (which have been considered for the M4T implementation), great relevance is assumed by flexibility and compliance with the Space Reference FOM.

As stated in 5.1, the proposed model-driven method makes use of a Data Types Library that aims at ensuring a clear separation of concerns between federation-related concepts and reference domain-related concepts.

In this respect, this section introduces the Space FOM Datatype Library, which has been designed in order to provide an implementation of the several items and data types specified by the SISO Space Reference FOM. The library is used during the refinement of the SysML model, in order to classify objects and interactions according to the taxonomy provided by the Space Reference FOM, and for typing attributes playing an active role in the HLA federation (e.g., model elements stereotyped as «objectattribute» or «interactionparameter»).

The Data Types Library has been structured in two different packages: the MIM package, which includes the datatypes specified by the HLA Standard MIM (MOM and Inizialization Module), and the SpaceFOM package, which provides data types, objects and interactions specified by the Space Reference FOM. As an example, datatypes and objects included in the SpaceFOM package are shown in Figure 5 and Figure 6, respectively.

\subsection{M4T: Model-To-Text Transformation Tool}

The automated generation of FOM modules from a SysML representation of a HLA federation is one of the most important objectives of the HRAF Engineering Framework. In this respect, beyond the refinement and the contextualization of the method at the basis of the proposed development process, this paper's contribution also includes the design and implementation of M4T, the transformation tool implementing the required model-to-text transformation. 


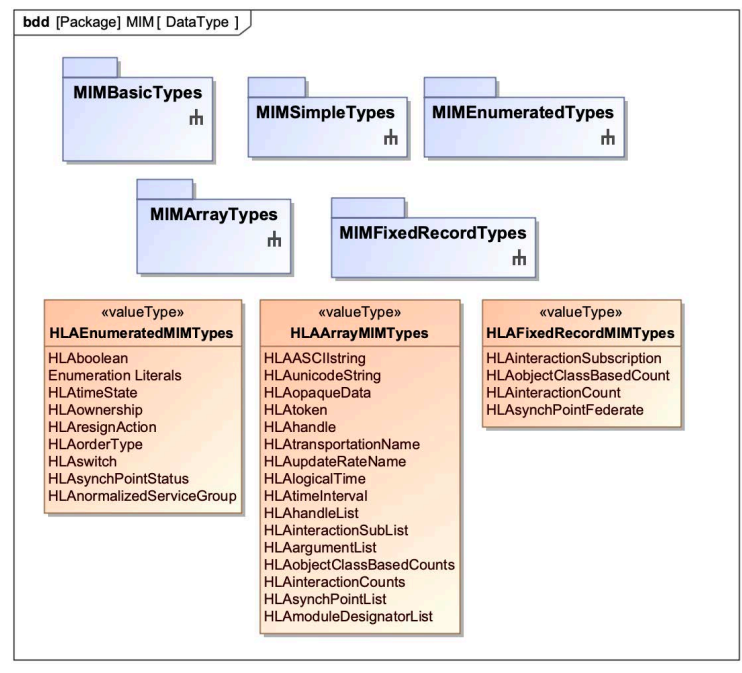

Figure 5: Datatypes Library: MIM Datatypes.

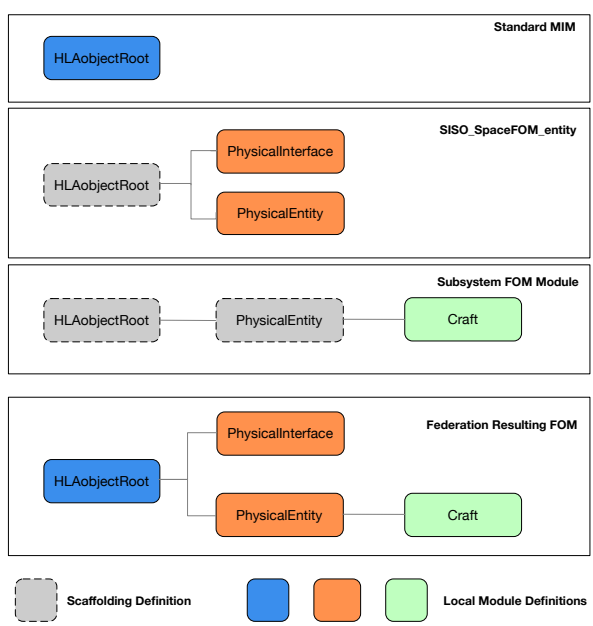

Figure 7: Modular FOM.

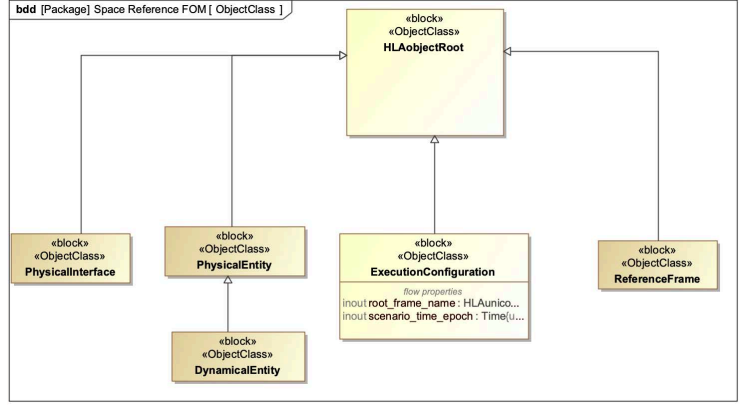

Figure 6: Datatypes Library: Space FOM Object Classes.

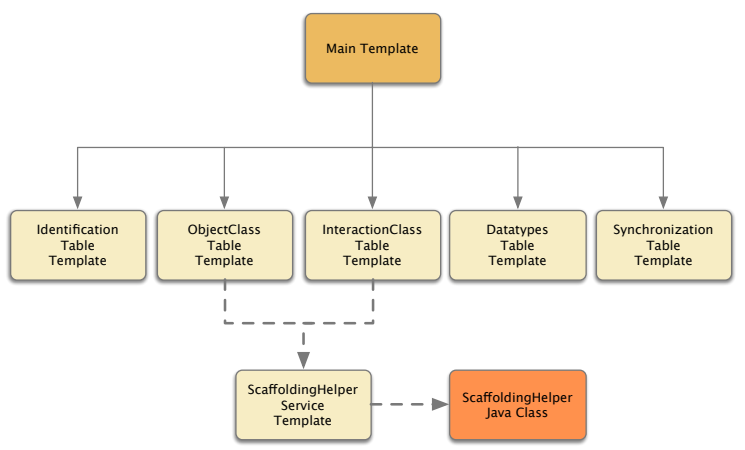

Figure 8: Architecture of M4T.

According to the MOF M2T (OMG 2008) standard, the M4T architecture, which is shown in Figure 8, is structured in different templates: the main template is responsible of the whole transformation and contains the logic required to activate the execution of the remaining four private templates, according to the structure of the input SysML model. Each template has been specified so as to generate one of the tables composing the FOM. A fifth template has also been specified in order to invoke the ScaffoldingHelper Java class, which handles the chain of inheritance relationships among model elements, which are too complex to be implemented by using the Acceleo language.

To better clarify the rationale of this design choice, it should be underlined that a requirement of the transformation is the generation of a modular FOM, as shown in Figure 7.

To ensure consistency among the several model elements specified in each FOM module, the composition makes use of a scaffolding mechanism: when a module includes a declaration of an element which extends an existing element specified in a different module, an empty placeholder (i.e., the scaffolding definition) is introduced. According to the example shown in Figure 7, the Craft objectclass in the Subsystem FOM Module extends the PhysicalEntity, which is specified in the SISO_SpaceFOM module. In turn, the 
PhysicalEntity is an extension of the HLAObjectRoot element, specified in the HLA standard MIM. The resulting FOM contains the complete specification of each element.

In order to handle the scaffolding, the M4T transformation has to cope with the several inheritance relationships which may exist between classes specified in different models (i.e., the SysML model and the Data Type Library). In Ecore, generalization is a one-way relationship and, as a consequence, a subclass is allowed to have direct access to its superclasses, but not the opposite.

Listing 1: ScaffoldingHelper Service Template.

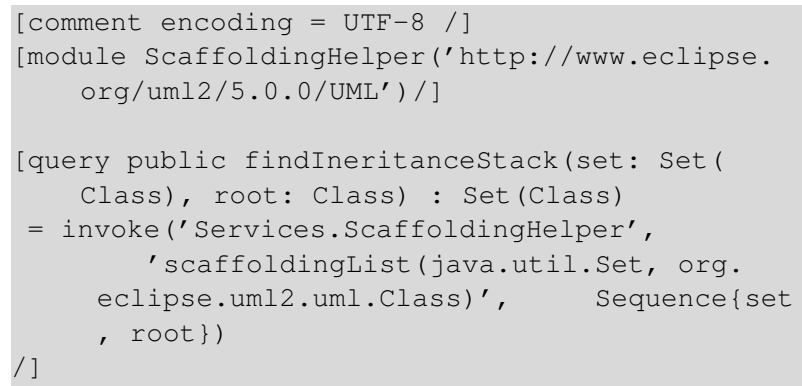

Listing 2: Fragment of ScaffoldingHelper Java Class.

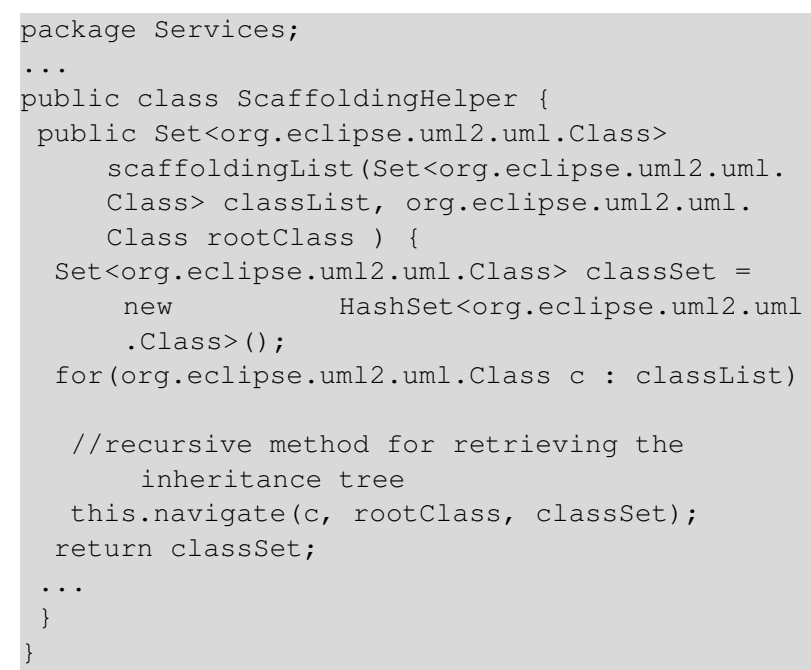

Indeed, a recursive Java method has been introduced to first determine a complete tree of inheritance relationships, and then generate the corresponding FOM module section from the superclass down to the related subclasses.

A fragment of the Service Template is shown in Listing 1, while a fragment of the ScaffoldingHelper class is shown in Listing 2.

\section{APPLICATION OF MODEL-DRIVEN HLA-DS EF TO HRAF}

In order to prove the effectiveness and the feasibility of the development process at the basis of the modeldriven HLA-DS Engineering Framework, and also to assess the correctness of M4T, the HRAF Project includes the investigation of the two scenarios introduced in Section 4. According to the HRAF activity plan, at the current state of the project, the method evaluation includes the generation of the FOM model and its system level validation by comparing the FOM generated using M4T with i) a manually specified FOM that is derived from the same mission-level federation model, and ii) the input SysML Federation model, so as to check completeness and consistency of the generated FOM. An inspection using comparison approach is proposed to qualitatively check whether the auto-generated FOM is a valid representation of the input model.

The SysML Federation model specified for the MSR and NEO scenarios is quite complex and a detailed description is out of the scope of this paper. To give an idea of the model complexity, Figure 9 shows the internal representation of the Guidance, Navigation and Control (GNC) subsystem, a component of the Craft systems. The latter is one of the systems which compose the Space segment of the Earth Return Orbiter (ERO) system. 
D’Ambrogio, Bocciarelli, Delfa and Kisdi

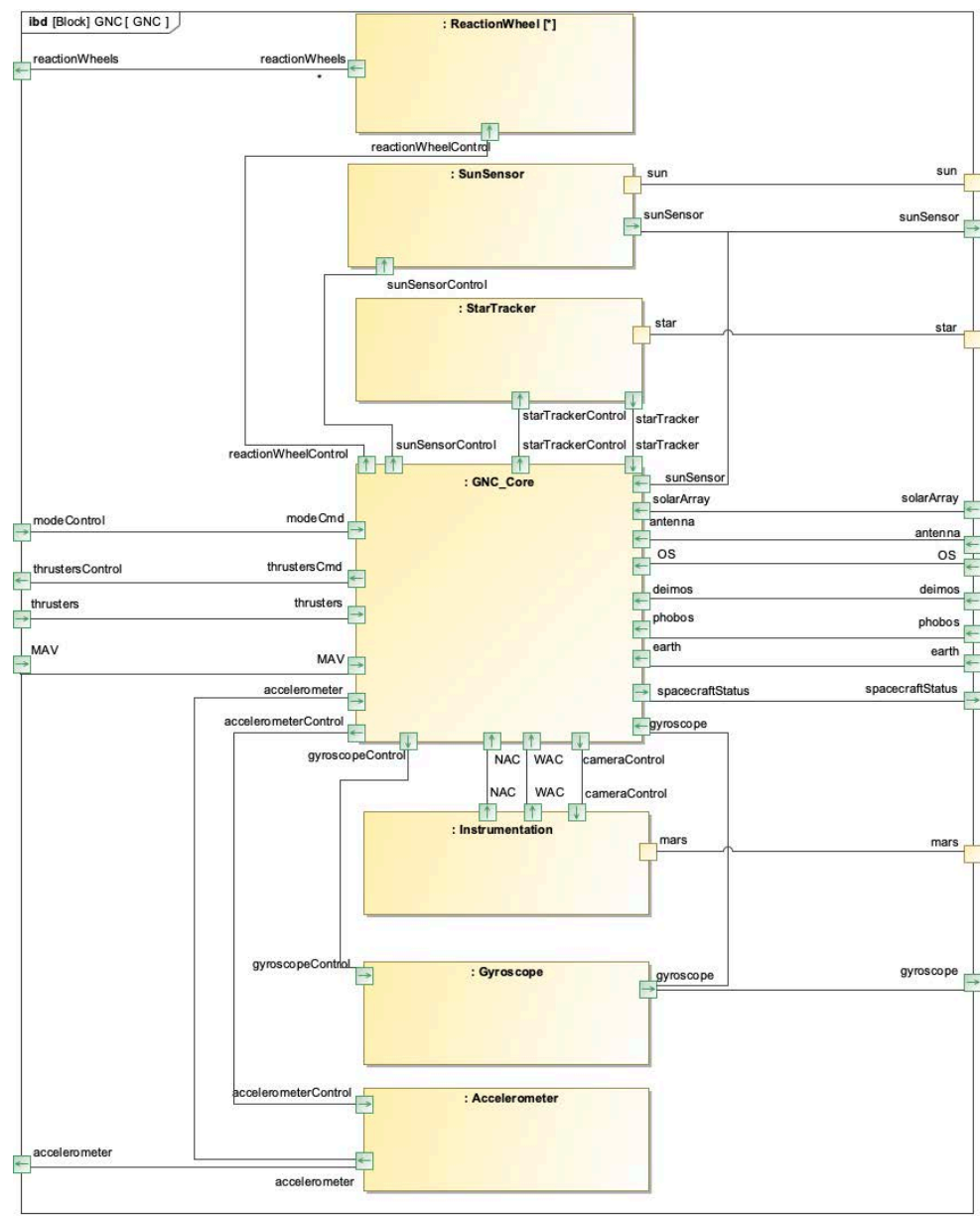

Figure 9: Internal Structure of the GNC.

In order to generate the FOM Model, the M4T transformation engine looks at those elements stereotyped by the FOM-HLA Profile and typed with elements from the Data Types Library. Each FOM module is generated according to the structure of those packages stereotyped as «Module». M4T considers the several elements stereotyped as «objectclass», «interactionclass» and «datatype». As an example, the MSR scenario includes the SysML block definition diagrams depicted in Figure 10, which also shows the attribute annotations by use of the «objectattribute» stereotype.

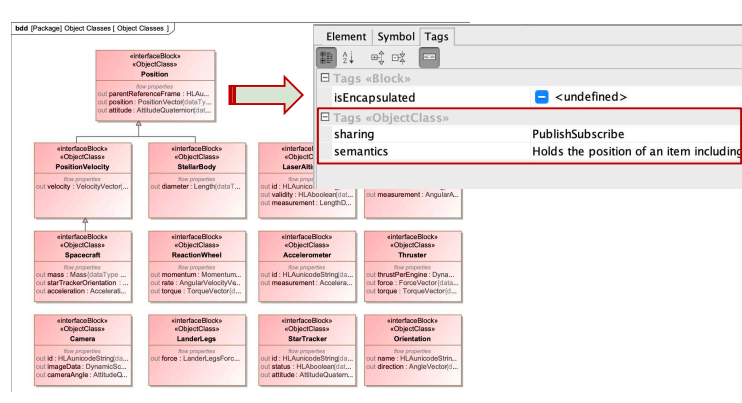

Figure 10: Federation Model (MSR Scenario) Object Classes.

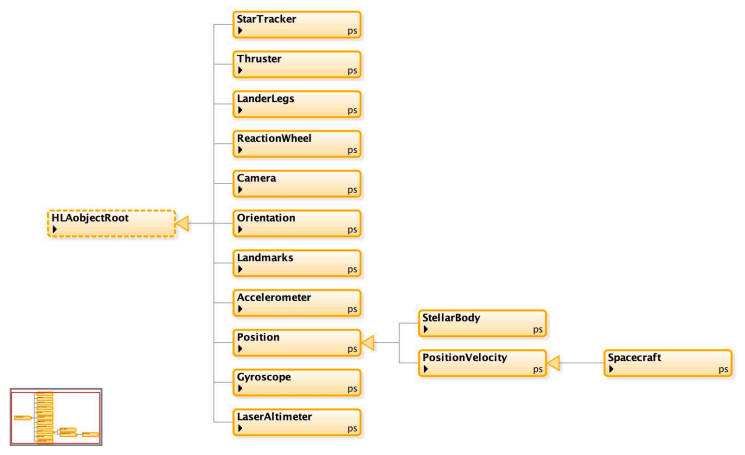

Figure 11: FOM - ObjectClass Table. 
The M4T execution with the MSR model given as input, produces as output one single FOM module. The MSR Federation FOM is obtained by the composition of such a module together with the following list of existing modules:

- the list of modules composing the SISO Space Reference FOM;

- the HLA Standard MIM FOM.

As an example of the resulting FOM, Figure 11 shows the Object Table.

The preliminary validation carried out by comparison with the manually specified FOM and by inspection of the source model has confirmed the consistency of the generated FOM.

\section{CONCLUSIONS}

In the context of the ESA HRAF program for the planetary exploration missions, this work has presented the result of a study, aiming at introducing model-driven techniques to support the HRAF HLA-DS Engineering Framework. Specifically, this work has proposed a model-driven development process that exploits methods and tools at the core of the OMG's MDA, and presents M4T, a model-to-text transformation tool, for generating FOM modules from a SysML specification of the federation under study. At the current stage, the proposed approach has been successfully evaluated to generate the required FOM modules for two scenarios of the HRAF exploration. The auto-generated FOM proved to be correct by comparison to the input model and to the FOM manually specified starting from the same mission-level federation model. The validation confirmed that the proposed approach effectively supports ESA objectives. Future work includes an extensive V\&V of the model-driven HLA-DS EF, including the comparison between the results obtained by an auto-generated full-fledged federation and the ones obtained by the related manually implemented federation.

\section{REFERENCES}

Bocciarelli, P., A. D'Ambrogio, and G. Fabiani. 2012. "A Model-driven Approach to Build HLA-based Distributed Simulations from SysML Models". In Proceedings of the 2nd International Conference on Simulation and Modeling Methodologies, Technologies and Applications, SIMULTECH'12, pp. 49-60.

Bocciarelli, P., A. D’ Ambrogio, A. Falcone, A. Garro, and A. Giglio. 2015. "A model-driven approach to enable the distributed simulation of complex systems". In Proceedings of the 6th International Conference on Complex Systems Design and Management, CSDM. Paris, France.

Bocciarelli, P., A. D’Ambrogio, A. Giglio, and E. Paglia. 2019, April. "Automated Generation of FOM Modules for HLA-Based Distributed Simulations". In 2019 Spring Simulation Conference (SpringSim), pp. 1-12.

Cetinkaya, D., A. Verbraeck, and M. D. Seck. 2011. "MDD4MS: a model driven development framework for modeling and simulation". In Proceedings of the 2011 summer computer simulation conference, $\mathrm{pp}$. $113-121$.

Eclipse Foundation 2015. "Acceleo”. Website: https://eclipse.org/acceleo/.

Eclipse Foundation 2019. "Papyrus". Website: https://www.eclipse.org/papyrus/.

IEEE 2010a. 1730-2010 Distributed Simulation Engineering and Execution Process (DSEEP).

IEEE 2010b. IEEE 1516 - Standard for Modeling and Simulation High Level Architecture - Framework and Rules. 
IEEE 2010c. IEEE 1516.2- Standard for Modeling and Simulation High Level Architecture - Object Model Template (OMT) Specification.

Jain, A., R. Fujimoto, J. Crittenden, M. Liu, J. Kim, and Z. Lu. 2015. "Towards automating the development of federated distributed simulations for modeling sustainable urban infrastructures". In Winter Simulation Conference (WSC), 2015, pp. 2668-2679. IEEE.

Kapos, G.-D., V. Dalakas, A. Tsadimas, M. Nikolaidou, and D. Anagnostopoulos. 2014. "Model-based system engineering using SysML: Deriving executable simulation models with QVT". In Systems Conference (SysCon), 2014 8th Annual IEEE, pp. 531-538. IEEE.

Möller, B., E. Z. Crues, D. Dexter, A. Garro, A. Skuratovskiy, and A. Vankov. 2016. "A First Look at the Upcoming SISO Space Reference FOM”. In SISO 2016 Simulation Innovation Workshop (SIW).

NoMagic 2019. "Magic Draw". Website: https://www.magicdraw.com/main.php.

OMG 2008. MOF Model to Text Transformation Language. https://www.omg.org/spec/MOFM2T.

OMG 2014. MDA Guide, version 2.0.

OMG 2015. XML Metadata Interchange, version 2.5.1.

OMG 2017. System Modeling Language. https://www.omg.org/spec/SysML/1.5/.

Özhan, G., and H. Oguztüzün. 2015. "Transformation of Conceptual Models to Executable High-Level Architecture Federation Models". In Concepts and Methodologies for Modeling and Simulation, pp. 135-173. Springer.

Pitch 2019. "Pitch Developer Studio". Website: http://pitchtechnologies.com/products/developerstudio/.

Topçu, O., U. Durak, H. Oğuztüzün, and L. Yilmaz. 2016. Distributed Simulation: A Model Driven Engineering Approach. Springer.

\section{AUTHOR BIOGRAPHIES}

ANDREA D'AMBROGIO is associate professor of systems and software engineering at the Department of Enterprise Engineering of the University of Roma Tor Vergata (Italy). His research interests are in the areas of system performance and dependability engineering, model-driven systems and software engineering, business process management, and distributed simulation. His email address is dambro@uniroma2.it.

PAOLO BOCCIARELI is a postdoc researcher at the Department of Enterprise Engineering of the Uniersity of Roma Tor Vergata (Italy). His research interests include software and systems engineering, business process management, model-driven development and distributed simulation. His email address is paolo.bocciarelli@uniroma2.it.

JUAN DELFA is a senior system engineer with with an extended experience in the Aerospace, Robotics and Computer Science. As a member of the Exploration Preparation, Research and Technology team at ESA-ECSAT, he is the supervisor of the HRAF project family. His email address is Juan.Delfa@esa.int.

ARON KISDI is an engineer at the GMV Innovating Solutions at Harwell Campus. He does research in Autonomy and Robotics and he is the GMV leader for the Harwell Robotics and Autonomy Facility (HRAF). His email address is akisdi@gmv.com. 